\title{
Neural Correlates of Knee Extension and Flexion Force Control: A Kinetically-Instrumented Neuroimaging Study
}

\author{
Dustin R. Grooms ${ }^{1,2,3 *}$, Cody R. Criss ${ }^{1,4}$, Janet E. Simon ${ }^{1,2}$, Adam L. Haggerty ${ }^{1,2}$ \\ and Timothy R. Woh/ ${ }^{5,6}$
}

${ }^{1}$ Ohio Musculoskeletal and Neurological Institute, Ohio University, Grover Center, Athens, OH, United States, ${ }^{2}$ Division of Athletic Training, School of Applied Health Sciences and Wellness, College of Health Sciences and Professions, Ohio University, Grover Center, Athens, $\mathrm{OH}$, United States, ${ }^{3}$ Division of Physical Therapy, School of Rehabilitation and Communication Sciences, College of Health Sciences and Professions, Ohio University, Grover Center, Athens, OH, United States, ${ }^{4}$ Translational Biomedical Sciences Program, Heritage College of Osteopathic Medicine, Ohio University, Athens, $\mathrm{OH}$, United States, ${ }^{5}$ Honors Tutorial College, Ohio University, Athens, OH, United States, ${ }^{6}$ Division of Physical Therapy, School of Health and Rehabilitation Sciences, Ohio State University, Columbus, $\mathrm{OH}$, United States

OPEN ACCESS

Edited by: Taiar Redha,

Université de Reims

Champagne-Ardenne, France

Reviewed by:

Roxana G. Burciu,

University of Delaware, United States

Nejc Sarabon,

University of Primorska, Slovenia

*Correspondence:

Dustin R. Grooms

groomsd@ohio.edu

Specialty section: This article was submitted to Motor Neuroscience,

a section of the journal Frontiers in Human Neuroscience

Received: 28 October 2020 Accepted: 23 December 2020

Published: 04 February 2021

Citation:

Grooms DR, Criss CR, Simon JE,

Haggerty AL and Wohl TR

(2021) Neural Correlates of Knee

Extension and Flexion Force Control:

A Kinetically-Instrumented

Neuroimaging Study.

Front. Hum. Neurosci. 14:622637.

doi: 10.3389/fnhum.2020.622637
Background: The regulation of muscle force is a vital aspect of sensorimotor control, requiring intricate neural processes. While neural activity associated with upper extremity force control has been documented, extrapolation to lower extremity force control is limited. Knowledge of how the brain regulates force control for knee extension and flexion may provide insights as to how pathology or intervention impacts central control of movement.

Objectives: To develop and implement a neuroimaging-compatible force control paradigm for knee extension and flexion.

Methods: A magnetic resonance imaging (MRI) safe load cell was used in a customized apparatus to quantify force $(\mathrm{N})$ during neuroimaging (Philips Achieva 3T). Visual biofeedback and a target sinusoidal wave that fluctuated between 0 and $5 \mathrm{~N}$ was provided via an MRI-safe virtual reality display. Fifteen right leg dominant female participants (age $=20.3 \pm 1.2$ years, height $=1.6 \pm 0.10 \mathrm{~m}$, weight $=64.8 \pm 6.4 \mathrm{~kg}$ ) completed a knee extension and flexion force matching paradigm during neuroimaging. The force-matching error was calculated based on the difference between the visual target and actual performance. Brain activation patterns were calculated and associated with force-matching error and the difference between quadriceps and hamstring forcematching tasks were evaluated with a mixed-effects model $(z>3.1, p<0.05$, cluster corrected).

Results: Knee extension and flexion force-matching tasks increased BOLD signal among cerebellar, sensorimotor, and visual-processing regions. Increased knee extension forcematching error was associated with greater right frontal cortex and left parietal cortex activity and reduced left lingual gyrus activity. Increased knee flexion force-matching error was associated with reduced left frontal and right parietal region activity. Knee flexion 
force control increased bilateral premotor, secondary somatosensory, and right anterior temporal activity relative to knee extension. The force-matching error was not statistically different between tasks.

Conclusion: Lower extremity force control results in unique activation strategies depending on if engaging knee extension or flexion, with knee flexion requiring increased neural activity (BOLD signal) for the same level of force and no difference in relative error. These fMRI compatible force control paradigms allow precise behavioral quantification of motor performance concurrent with brain activity for lower extremity sensorimotor function and may serve as a method for future research to investigate how pathologies affect lower extremity neuromuscular function.

Keywords: force sense, functional magnetic resonance imaging, quadriceps, hamstring, lower extremity, sensorimotor control

\section{INTRODUCTION}

Determining how the central nervous system regulates force is vital for understanding the neural control of biomechanical action. The integration of neuroimaging techniques with simultaneous biomechanical recording has allowed for concurrent capture of joint position and force with neural activity (Liu et al., 2000; Ward et al., 2008; Naufel et al., 2019). However, the majority of investigations have focused on the upper extremity and the primary motor cortex to elucidate the relationship between muscle force and neural activity (Georgopoulos et al., 1992; Ashe, 1997; Ward et al., 2008). Studies examining neural activity associated with lower extremity motor control have not quantified motor performance beyond movement timing (Luft et al., 2002; Kapreli et al., 2007; Grooms et al., 2019) or have been limited to electroencephalography paradigms, which provide excellent temporal resolution but lack the spatial resolution of functional magnetic resonance imaging (fMRI; Poortvliet et al., 2015). Prior work specific to fMRI has examined neural correlates of quadriceps force regulation in patients with knee osteoarthritis (Shanahan et al., 2015) using an isometric, force-matching paradigm, finding an anterior shift of the knee representation within the primary motor cortex in those with knee osteoarthritis. Various research groups have also employed cycle ergometers (Mehta et al., 2009), gait simulations (Jaeger et al., 2016), or leg press (Grooms et al., 2019) movement paradigms to quantify lower extremity movement with brain imaging. While these paradigms demonstrated success to activate the sensorimotor network and do so reliably, many fMRI lower extremity paradigms are metronome-paced and do not attempt to quantify motor performance (Luft et al., 2002; Kapreli et al., 2006). Therefore, the development of lower extremity paradigms that can concurrently measure neural activity via fMRI and biomechanical performance may offer more precise methods to investigate central strategies for force regulation, with implications for pathologies affecting sensorimotor control of the lower extremity (Hortobágyi et al., 2004; Ward et al., 2019).

Prior biomechanically isolated work has demonstrated force control deficits in a variety of orthopedic and neurological pathologies of the lower extremity (Hortobágyi et al., 2004;
Docherty and Arnold, 2008; Telianidis et al., 2014) but a clear brain-behavioral interaction has yet to be established (Baumeister et al., 2011). Further, no study to our knowledge has attempted to contrast how the brain regulates force when engaged in knee extension (quadriceps-dominant activity) relative to knee flexion (hamstring-dominant activity). Unique deficits in quadriceps and hamstring function have been reported in a variety of orthopedic and neurological conditions, and the restoration of respective muscle and joint function is vital for the recovery and resumption of activities of daily living, adequate mobility, and mitigating the development of chronic conditions such as osteoarthritis (Manini et al., 2007; Manini and Clark, 2012; Tourville et al., 2014; Arhos et al., 2020). As lower extremity pathologies have been found to manipulate both quadriceps and hamstring muscle activity, timing, and function, determining the neural mechanisms for each is vital to better understand how lower extremity motor control is centrally governed (Telianidis et al., 2014; Abourezk et al., 2017; Blackburn et al., 2017; Hohmann et al., 2019). Isolating neural correlates of quadriceps and hamstring force generation and control may highlight central mechanisms for function following injury and permit the development of novel therapies that restore function. Therefore, our purpose was to: (1) develop and test a lower extremity neuroimaging paradigm for knee extension and flexion force control to better understand how the nervous system regulates lower extremity forces; and (2) determine differences between knee extension and flexion neural activity during a force control task.

\section{MATERIALS AND METHODS}

\section{Participants}

This study was approved by Ohio University's Institutional Review Board and all participants signed the informed consent document. We included female recreational athletes (at least $3 \mathrm{~h}$ of moderate to vigorous exercise per week, including $1 \mathrm{~h}$ of running, cutting, pivoting, or decelerating every week) aged 18-30 years. This population was selected for the following investigative work as they are at unique increased risk for noncontact knee injuries, whereby during athletics, exercise, 
or activities of daily living that require rapid movement, sensorimotor control of the knee is compromised, resulting in positions that put excessive strain on the joint ligaments (Beynnon et al., 2014; Montalvo et al., 2019).

A sample size estimate was calculated based on effects reported by Shanahan et al. (2015) for the correlation to forceerror, and Trinastic et al. (2010) for the contrast between movement conditions. For the force-error correlate analysis, an $r=0.83$ was reported for the relationship between error and motor cortex peak activation location (Shanahan et al., 2015). A sample size estimate was calculated based on $r=0.83$, $\alpha=0.05$, and $1-\beta=0.8$ indicating a total sample size of 8 is required. For the motor condition analysis, the effect size between ankle plantarflexion and dorsiflexion was calculated as $d=1.42$ (Trinastic et al., 2010). A sample size estimate was calculated based on $d=1.42, \alpha=0.05$, and $1-\beta=0.8$ resulting in needing a sample size of 7 . Additionally, we modeled our study on previous literature of Newton et al. (2008) and Mehta et al. (2009) regarding paradigm development who enrolled 9 and 10 participants, respectively. Therefore, enrolling 15 participants provided adequate power for the proposed study. We enrolled 15 participants $(15 \mathrm{~F}$; age $=20.3 \pm 1.2$ years, height $=1.6 \pm 0.10 \mathrm{~m}$, and weight $=64.8 \pm 6.4 \mathrm{~kg}$ ) in this study. All participants were right leg dominant and met the exercise requirement criteria, as determined by the Marx Activity Rating Scale (Table 1; Marx et al., 2001).

We excluded participants who were contraindicated for fMRI (e.g., pregnancy, implanted metal devices, claustrophobia, and any other criteria as determined by the MRI operator), have a visual impairment, have a history of seizures or epilepsy, or have a history of surgery on the back, hip, leg, knee, etc. Other screening criteria included: primary sport, leg dominance, previous leg injury, medical history anxiety disorder, ADHD, depression, diabetic neuropathy, concussion or traumatic brain injury, cerebral palsy, balance disorder, vertigo, Parkinson's disease, multiple sclerosis, substance abuse or dependence, heart disease/defect, and prescription medication use within the $24 \mathrm{~h}$ before data collection. No individuals reported any of the previous medical conditions or consumed any medications impacting the data collection.

\section{fMRI Data Collection}

Data collection was completed in a single neuroimaging session ( $\sim 45$ min including set-up, instruction, and scan time). During imaging, all participants wore standardized shorts and

\section{TABLE 1 | Demographics and force error.}

\begin{tabular}{lc}
\hline Data & Mean \pm SD \\
\hline Age (years) & $20.3 \pm 1.2$ \\
Height (m) & $1.6 \pm 0.10$ \\
Weight (kg) & $64.8 \pm 6.4$ \\
Activity level (Marx) & $9.93 \pm 5.50$ \\
$\quad$ Run & $3.00 \pm 0.85$ \\
Cut & $2.07 \pm 1.62$ \\
Decelerate & $2.40 \pm 1.64$ \\
Pivot & $2.27 \pm 1.49$ \\
Knee extension error $(N)$ & $1.068 \pm 0.327$ \\
Knee flexion error $(N)$ & $0.999 \pm 0.189$
\end{tabular}

socks without shoes to reduce the possibility of altered skin tactile feedback. Participants also wore a splint to lock their right (dominant leg) ankle at neutral $\left(\sim 90^{\circ}\right)$ to minimize ankle movement throughout the scan. Headphone and hearing protection was provided for subject comfort and safety and to facilitate communication during scanning. While lying supine in the fMRI scanner, participants were strapped down to the table with four straps, one across the thighs at the mid-point between the greater trochanter and knee joint line, one across the hips at the anterior superior iliac spines, and two across the chest, from each shoulder to the pelvis at the iliac crest. The knee was fixed near terminal extension between $10^{\circ}$ and $15^{\circ}$ of flexion. Participants were also fitted with customized padding to reduce head motion. This padding was high-density MRI-safe foam that was inserted around the sides and top of the head to remove space between the skull and head coil. This was customized based on skull size, with those with larger skulls requiring less padding and smaller skulls requiring more padding.

fMRI scans were collected with a 16-channel head coil. Before the functional data collection, a three-dimensional high-resolution T1-weighted image (repetition time (TR): 2,000 ms, echo time (TE): $4.58 \mathrm{~ms}$, field of view: $256 \times 256 \mathrm{~mm}$; matrix: $256 \times 256$; slice thickness $1 \mathrm{~mm}, 176$ slices, $8^{\circ}$ flip-angle) was collected for image registration $(\sim 8 \mathrm{~min})$. fMRI collection parameters include 10 whole-brain gradient-echo-echo planar scans per block (four force-matching blocks, five rest blocks) acquired with a $3 \mathrm{~s}$ TR with anterior-posterior phase encoding and a $3.75 \times 3.75$ in-plane resolution, $5 \mathrm{~mm}$ slice thickness for 38 axial slices with a $35 \mathrm{~ms} \mathrm{TE}, 90^{\circ}$ flip angle, the field of view $240 \mathrm{~mm}$ and $64 \times 64$ matrix. Each functional forcematching run lasted $4 \mathrm{~min}$ and $30 \mathrm{~s}$. fMRI measured regional brain activity during rest and motor control conditions, which were contrasted to isolate the regional brain activity to the isometric knee extension and flexion force-matching tasks.

The isometric force-matching motor task required the participant to either "kick up" or "press down" against a load cell (Biopac Systems Inc., TSD121B-MRI, 1,000 Hz sampling frequency) at the ankle (Figure 1C). Both knees rested upon a foam roller, while only the dominant, right leg was additionally strapped to a device against the load cell. Participants had to match their force output (visualized with biofeedback provided by MRI-safe virtual reality) with a sine wave that oscillated $(1.2 \mathrm{~Hz})$ from 0 to $5 \mathrm{~N}$ for $30 \mathrm{~s}$ with $30 \mathrm{~s}$ of rest for four total cycles, resulting in four force-matching blocks interspersed with five rest blocks of $30 \mathrm{~s}$ each (with the paradigm starting and ending with rest) for a total run time of $4 \mathrm{~min}$ and $30 \mathrm{~s}$ (Figures 1A,B). Standardized auditory cues informed participants when to begin and end force-matching. The force-matching error was calculated based on the difference between the visual target (sine wave) and actual performance (biofeedback). The force level for this study was low and we recruited a young active cohort to minimize the potential influence of fatigue; however, fatigue was monitored regularly and breaks were offered. No participants indicated fatigue or needed a break beyond the few minutes between scans.

Participants practiced the force-matching task for a full run with immediate examiner feedback if instructions were not 


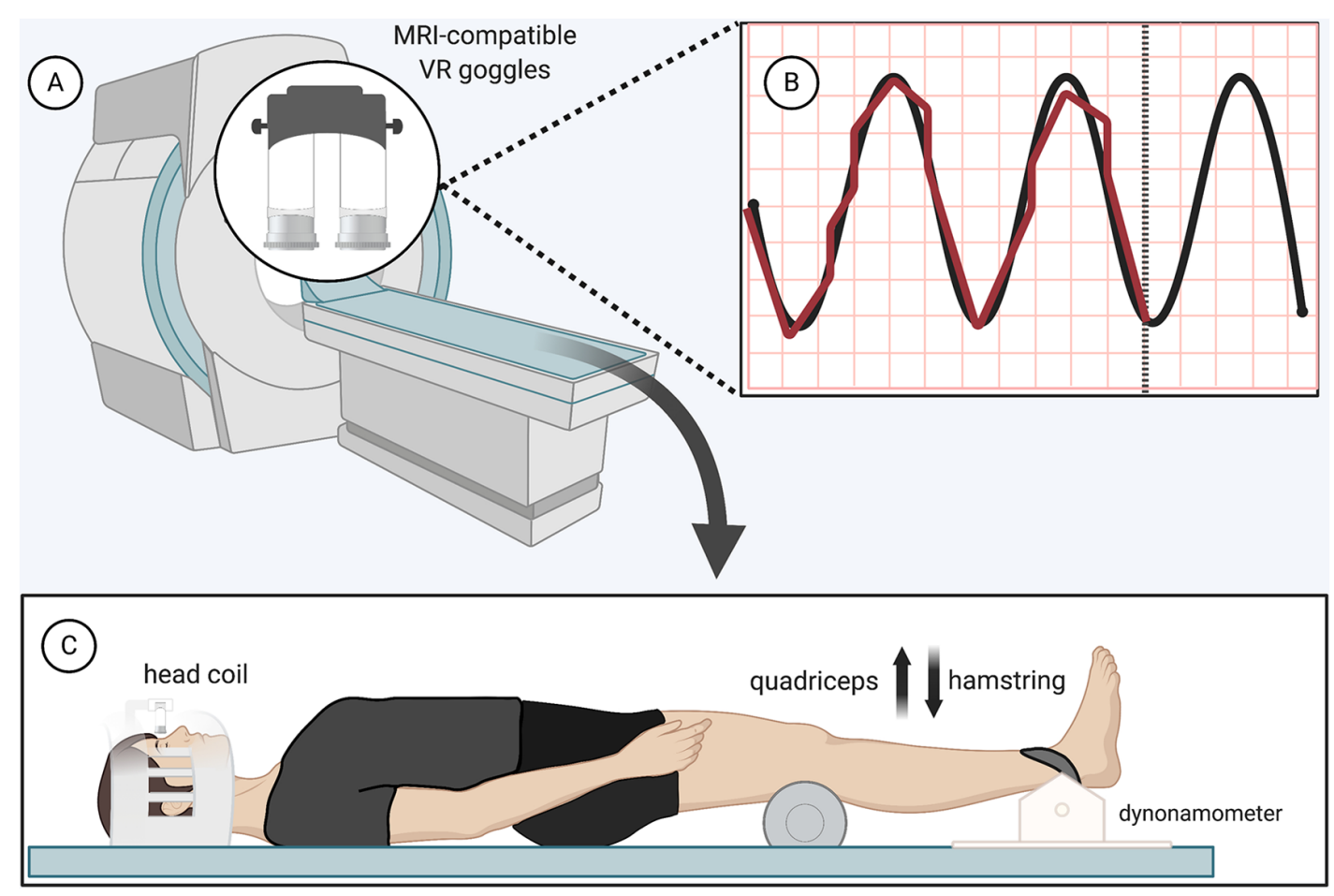

FIGURE 1 | Functional magnetic resonance imaging (fMRI) force-matching task-(A) magnetic resonance imaging (MRI)-compatible virtual reality display (B) black, Sinewave graph $(0-5 \mathrm{~N}, 1.2 \mathrm{~Hz})$; red, real-time display of participant force $\mathbf{( C )}$ load cell apparatus and patient position (participant restraints not pictured). Created with Biorender.

understood before completing the task during scanning. Also before data collection at the MRI, participants completed a mock MRI session where they familiarized themselves with the MRI environment, restraints to reduce head motion, and the lower extremity motor task. The participants were permitted to ask questions and practice the tasks with feedback from the experimenter. The practice session included three practice blocks (30 s each) of each force-matching task with examiner cueing to ensure the participant understood the task, followed by a complete run of each task with the same feedback and timing as during the actual MRI data collection session.

\section{Error Calculation and Statistical Analysis}

The force-matching error was recorded continuously throughout the force-matching tasks. For statistical analysis, error across the 30-s blocks was partitioned into 3-s intervals (the time interval for one sine wave). The first 3-s interval of each 30-s block was removed from the data analysis, as participants commonly required a few seconds to become acclimated to the task during the initiation of the movement block and thus, was shown to bias the overall average of the remaining nine intervals. The average error for each block was determined by the root mean square of the differential from target force to actual force on the remaining nine sets of 3-s intervals within each block, and the average error across the four blocks was computed for each participant for statistical analyses. Average knee extension and flexion force-matching error were compared with a paired samples $t$-test with an alpha set at 0.05 .

\section{fMRI Data and Statistical Analysis}

The fMRI technique used in this study quantified the bloodoxygen-level-dependent (BOLD) signal via the hemodynamic response by contrasting the respective force-matching condition with interspersed rest conditions (Friston et al., 1995). We controlled for the additional sensory feedback of the strap across the shank by ensuring it was tightly pressed during both the rest and force-matching conditions, but the pressure of this tactile stimulus unavoidably changes with contraction and may thus contribute to the overall BOLD response. The BOLD response, quantified via fMRI collection and analysis, has been validated against direct neural recordings, demonstrating a very high correlation between blood flow and neural activity (Logothetis et al., 2001; Goense and Logothetis, 2008). The reliability of fMRI quantification of the BOLD signal is generally high and specific to knee movement and has high inter-session reliability (Newton et al., 2008; McGregor et al., 2012).

The fMRI statistical analyses were performed using the Oxford Centre for Functional MRI of the Brain Software Library (Smith et al., 2004; Jenkinson et al., 2012). Image analysis began with standard pre-statistic processing applied to individual data in the standardized FSL recommended order (Jenkinson et al., 2012), which included nonbrain removal, slice timing correction, standard motion correction, and realignment 
parameters (three rotations and three translations) as covariates to limit confounding effects of head movement and spatial smoothing at $6 \mathrm{~mm}$ before statistical analysis (Jenkinson et al., 2002). One participant was removed from the knee extension force-matching analysis due to excessive head motion (>0.5 $\mathrm{mm}$ ) and two removed from the knee flexion force-matching analysis, resulting in $n=14$ for knee extension, $n=13$ for knee flexion, and $n=13$ for comparison between knee extension and knee flexion. High-pass temporal filtering at $90 \mathrm{~Hz}$ and time-series statistical analyses were carried out using a linear model with local autocorrelation correction. Functional images were co-registered with the respective high-resolution T1 image and the standard Montreal Neurological Institute template 152 using linear image registration. This registration process allowed data from each participant to be spatially aligned on a standardized brain template for comparison.

The subject-level analysis of knee sensorimotor control relative to rest was completed using a $z$ score greater than 3.1 and a (corrected) cluster significance threshold of $\alpha<0.05$. The cluster correction for multiple comparisons uses a variant of the Gaussian random field theory to decrease type I error in the statistical parametric mapping of imaging data by evaluating the activation not only at each voxel but also at the surrounding voxel cluster (as it is unlikely that the voxel tested and surrounding voxels are active above the threshold due to chance; Poldrack et al., 2011). The paired contrast between each individual's quadriceps vs. hamstring force control neural activity was performed with group $z$ statistic images set at a threshold of $z$ scores of greater than 3.1 and a corrected cluster significance level of $\alpha<0.05$. As this was a brain activity correlate identification study, the effect size ( $r$-value) of the relationship between brain activity and behavior are not reported to avoid circularity (voxel selection and magnitude estimation on the same data) and a follow-up validation study is required to estimate effect size with the identified regions from this work (Kriegeskorte et al., 2009, 2010).

\section{RESULTS}

Regional brain activation is reported as contralateral [indicating activation on the opposite side of the task, or the left hemisphere, as the task was always completed with the right (dominant) lower extremity] or ipsilateral (being the same side as the task, or the right hemisphere; Tables 2-4). Regions of brain activity are reported that were identified in FSLeyes based on peak-voxel with the Harvard-Oxford Cortical and Subcortical Structural Atlas (Desikan et al., 2006), Juelich Histological Atlas (Eickhoff et al., 2006, 2007) and the Cerebellar Atlas in MNI152 space after normalization with FNIRT (Diedrichsen et al., 2009) and with FSL tool atlasquery (Jenkinson et al., 2012). The atlasquery function from FSL utilizes the averaged probability across all voxels in the cluster to identify probabilistic anatomy across the cluster ensuring reporting of peak voxel location and overall cluster spatial representation.

Both knee extension and flexion force-matching tasks elicited increased bilateral BOLD signal among cerebellar, sensorimotor, and visual-processing regions (Figures 2, 4).
Increased knee extension force-matching error was associated with increased BOLD signal within the ipsilateral frontal cortex and contralateral parietal cortex and decreased contralateral BOLD signal within the lingual gyrus and intracalcarine cortex (Figure 3). Increased knee flexion force-matching error was related to decreased contralateral frontal and ipsilateral parietal region activity (Figure 5). Knee flexion force control had increased bilateral premotor, secondary somatosensory, and right anterior temporal activity relative to knee extension force control (Figure 6). Force-matching error performance was not statistically different between the knee extension and flexion tasks (Table 1). Head motion during the knee extension task was: $0.28 \pm 0.17 \mathrm{~mm}$ absolute motion and $0.11 \pm 0.10 \mathrm{~mm}$ relative motion. Head motion during the knee flexion task was: $0.22 \pm 0.13 \mathrm{~mm}$ absolute motion and $0.10 \pm 0.09 \mathrm{~mm}$ relative motion.

\section{DISCUSSION}

Lower extremity force control results in unique neural activation strategies depending on if engaging the quadriceps for knee extension or the hamstrings for knee flexion, with knee flexion requiring more sensorimotor neural activity for the same level of force generation and relative error. This paradigm allows precise behavioral quantification of motor performance concurrent with brain activity for lower extremity sensorimotor function, which may serve as a method for future research to investigate how pathologies or interventions affect lower extremity neuromuscular function.

\section{Neural Correlates of Knee Extension Force Control}

Knee extension force-matching had a neural activation pattern similar to prior reports of lower extremity knee-focused and quadriceps-dominant movements, with activation across the cortical and subcortical sensorimotor network (Luft et al., 2002; Kapreli et al., 2007). Quadriceps force error was associated with increased activity in frontal and parietal regions and associated with decreased crossmodal (Calvert, 2001) region activity (intracalcarine cortex and lingual gyrus) along the border of the occipital and parietal cortex.

Increased activation of frontal regions with increased error could indicate force control is more complex for those with a higher force-matching error, as previous research has identified an association between increased frontal activity with increased task complexity error (Schubotz and von Cramon, 2002; Mehta et al., 2012; Dunst et al., 2014). It is also possible that as a participant began to perform poorly and visualize their error, they engaged in more extensive or rapid recalibration to attempt to remain on target, requiring greater levels of attentive neural processing (Tracy, 2007; Tracy et al., 2007; Baweja et al., 2009). However, despite increased neural activity among attention and executive function-related brain regions, the relative error was higher which could also be simply a byproduct of more actively attending to their mismatched biofeedback and not secondary to employing a strategy to correct it (Tracy, 2007). 
TABLE 2 | Regions of increased brain activity during the knee extension force-matching task.

\begin{tabular}{|c|c|c|c|c|c|c|c|}
\hline \multirow[t]{2}{*}{ Cluster index } & \multirow[t]{2}{*}{ Brain regions } & \multirow[t]{2}{*}{ Voxel count } & \multirow[t]{2}{*}{$P$-value } & \multicolumn{3}{|c|}{ Peak MNI voxel } & \multirow[t]{2}{*}{ Z stat-max } \\
\hline & & & & $x$ & $y$ & $\mathbf{z}$ & \\
\hline \multicolumn{8}{|c|}{ Overall activation during knee extension force-matching } \\
\hline 6 & $\begin{array}{l}\text { B Precentral gyrus, Postcentral gyrus, Superior parietal lobule, } \\
\text { Lateral occipital cortex }\end{array}$ & 17,662 & $<0.00001$ & 0 & -34 & 56 & 10.1 \\
\hline 5 & B Precentral gyrus, Corticospinal tract, R Thalamus & 1,567 & $<0.00001$ & 10 & -16 & 4 & 4.99 \\
\hline 4 & Corticospinal tract, L Thalamus & 1,137 & $<0.00001$ & -8 & -18 & 16 & 5.57 \\
\hline 3 & Precentral Gyrus, Inferior frontal gyrus, Premotor cortex & 375 & 0.000116 & -56 & 0 & 38 & 7.22 \\
\hline 2 & R Cerebellum VIIIA, VIIB, IX & 284 & 0.000882 & 32 & -50 & -48 & 4.79 \\
\hline 1 & L Cerebellum VIIB, VIIIA, VIIIB, IX & 182 & 0.0113 & -20 & -70 & -44 & 6.26 \\
\hline \multicolumn{8}{|c|}{ Neural activity increase associated with knee extension force error } \\
\hline 3 & L Postcentral gyrus, Superior parietal lobule & 206 & 0.00598 & -20 & -40 & 76 & 4.89 \\
\hline 2 & R Frontal pole & 142 & 0.0344 & 30 & 52 & 20 & 5.02 \\
\hline 1 & R Middle frontal gyrus & 130 & 0.0489 & 46 & 12 & 40 & 4.24 \\
\hline \multicolumn{8}{|c|}{ Neural activity decrease associated with knee extension force error } \\
\hline 1 & Intracalcarine cortex, Lingual gyrus & 161 & 0.0201 & -14 & -82 & 10 & 4.68 \\
\hline
\end{tabular}

Regions of brain activity are reported that were identified in FSLeyes with the Harvard-Oxford cortical and subcortical structural atlas, Julich histological atlas, and the Cerebellar atlas in MNI152 space after normalization with FNIRT by peak voxel and with FSL tool atlasquery. B, bilateral; L, left; R, right.

TABLE 3 | Regions of increased brain activity during the knee flexion force-matching task.

\begin{tabular}{|c|c|c|c|c|c|c|c|}
\hline \multirow[t]{2}{*}{ Cluster index } & \multirow[t]{2}{*}{ Brain regions } & \multirow[t]{2}{*}{ Voxel count } & \multirow[t]{2}{*}{$P$-value } & \multicolumn{3}{|c|}{ Peak MNI voxel } & \multirow[t]{2}{*}{ Z stat-max } \\
\hline & & & & $x$ & $y$ & $\mathbf{z}$ & \\
\hline \multicolumn{8}{|c|}{ Overall activation during knee flexion force-matching } \\
\hline 3 & $\begin{array}{l}\text { B Postcentral gyrus, Precentral gyrus, Superior parietal } \\
\text { lobule, Lateral occipital cortex, Supplementary motor } \\
\text { cortex, Cingulate gyrus }\end{array}$ & 16,647 & $<0.00001$ & -42 & -78 & -8 & 11.3 \\
\hline 2 & $\begin{array}{l}\text { B Precentral gyrus, Supramarginal gyrus, Lateral } \\
\text { occipital cortex, Lingual gyrus, Occipital fusiform gyrus, } \\
\text { Cerebellum Right I-V, VIIB, VIIIA, Left VIIB, VIIIA }\end{array}$ & 12,626 & $<0.00001$ & 24 & -70 & -56 & 10.3 \\
\hline 1 & R Frontal pole, Frontal orbital cortex & 189 & 0.008 & 26 & 34 & -22 & 4.91 \\
\hline \multicolumn{8}{|c|}{ Neural activity decrease associated with knee flexion force error } \\
\hline 2 & $\begin{array}{l}\text { R Precuneus, Postcentral gyrus, Posterior cingulate } \\
\text { gyrus, Superior parietal lobule }\end{array}$ & 257 & 0.00138 & 6 & -40 & 50 & 5.91 \\
\hline 1 & $\begin{array}{l}\text { L Frontal pole, Superior frontal gyrus, Middle frontal } \\
\text { gyrus }\end{array}$ & 215 & 0.00402 & -36 & 30 & 40 & 5.25 \\
\hline
\end{tabular}

Regions of brain activity are reported that were identified in FSLeyes with the Harvard-Oxford cortical and subcortical structural atlas, Julich histological atlas, and the cerebellar atlas in MNI152 space after normalization with FNIRT by peak voxel and with FSL tool atlasquery. There was no significant increased neural activity associated with knee flexion error. B, bilateral; L, left; $R$, right.

TABLE 4 | Regions of difference between knee extension and flexion force-matching.

\begin{tabular}{|c|c|c|c|c|c|c|c|}
\hline \multirow[t]{2}{*}{ Cluster index } & \multirow[t]{2}{*}{ Brain regions } & \multirow[t]{2}{*}{ Voxel count } & \multirow[t]{2}{*}{$P$-value } & \multicolumn{3}{|c|}{ Peak MNI voxel } & \multirow[t]{2}{*}{ Z stat-max } \\
\hline & & & & $\boldsymbol{x}$ & $y$ & $z$ & \\
\hline \multicolumn{8}{|c|}{ Increased neural activity knee flexion > knee extension force control } \\
\hline 6 & L Precentral and Postcentral gyrus & 741 & $<0.00001$ & -62 & -8 & 42 & 5.99 \\
\hline 5 & L Middle temporal gyrus, Angular gyrus, Inferior parietal lobule & 259 & 0.00112 & -62 & -52 & 16 & 5.19 \\
\hline 4 & R Temporal pole & 245 & 0.0016 & 44 & 22 & -34 & 5.55 \\
\hline 3 & R Superior temporal gyrus, Supramarginal gyrus, Middle temporal gyrus & 239 & 0.00187 & 48 & -38 & 2 & 5.16 \\
\hline 2 & L Supplementary motor cortex, Paracingulate gyrus & 194 & 0.00619 & -6 & 10 & 48 & 4.44 \\
\hline 1 & B Corticospinal tract, $L$ Thalamus & 134 & 0.0354 & -4 & -10 & -6 & 4.17 \\
\hline
\end{tabular}

Regions of brain activity are reported that were identified in FSLeyes with the Harvard-Oxford cortical and subcortical structural atlas, Julich histological atlas, and the cerebellar atlas in MNI152 space after normalization with FNIRT by peak voxel and with FSL tool atlasquery. B, bilateral; L, left; R, right.

By contrast, those with less force-matching error had increased crossmodal visual-spatial and somatosensory region processing (or increased error had decreased relative activity), which may be involved in aligning and maintaining visual feedback with force regulation from peripheral afferent signals to minimize discrepancy. Previous work within the upper extremity has identified the lingual gyrus and intracalcarine regions to respond to congruent visual and somatosensory feedback (crossmodal; Driver and Spence, 1998; Macaluso et al., 2000). Further, extrastriate activity in the lingual gyrus and intracalcarine cortex has been implicated to be involved in body perception, and active during both visual and limb movements (Astafiev et al., 2004). Therefore, increased extrastriatal activity may correspond with a superior ability to align visual stimuli with proprioceptive afferent signals to minimize force-matching discrepancy. However, the increased 


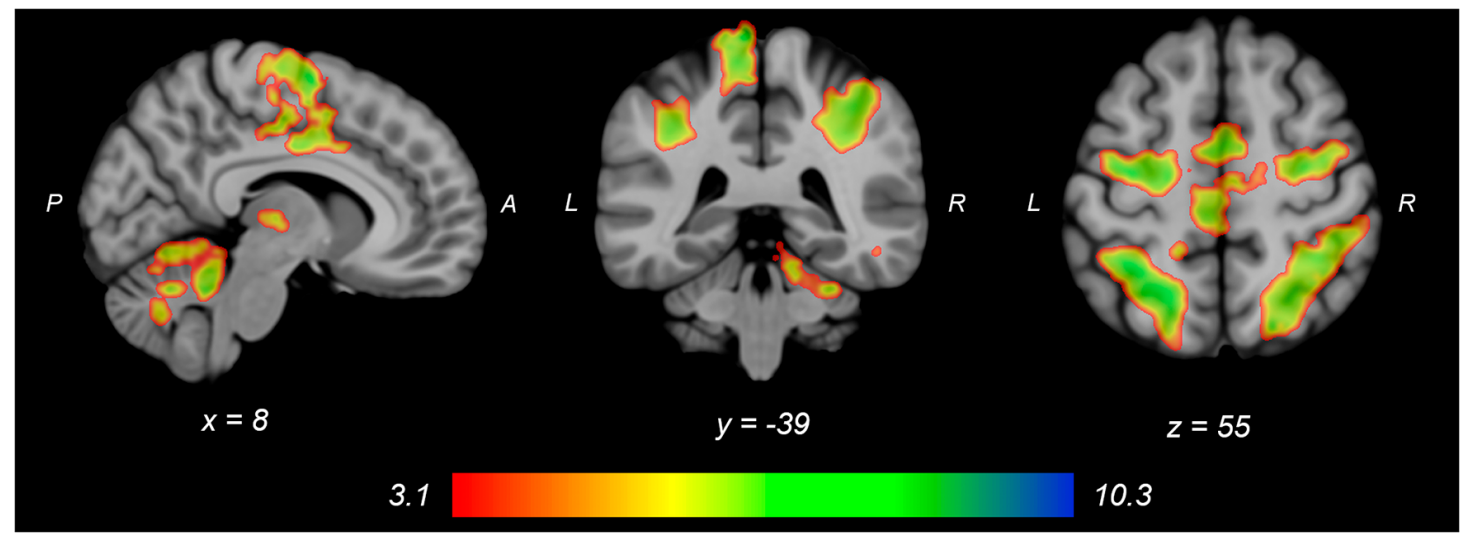

FIGURE 2 | Group average neural activity for knee extension force-matching from Table 2. P, posterior; A, anterior; L, left; R, right.

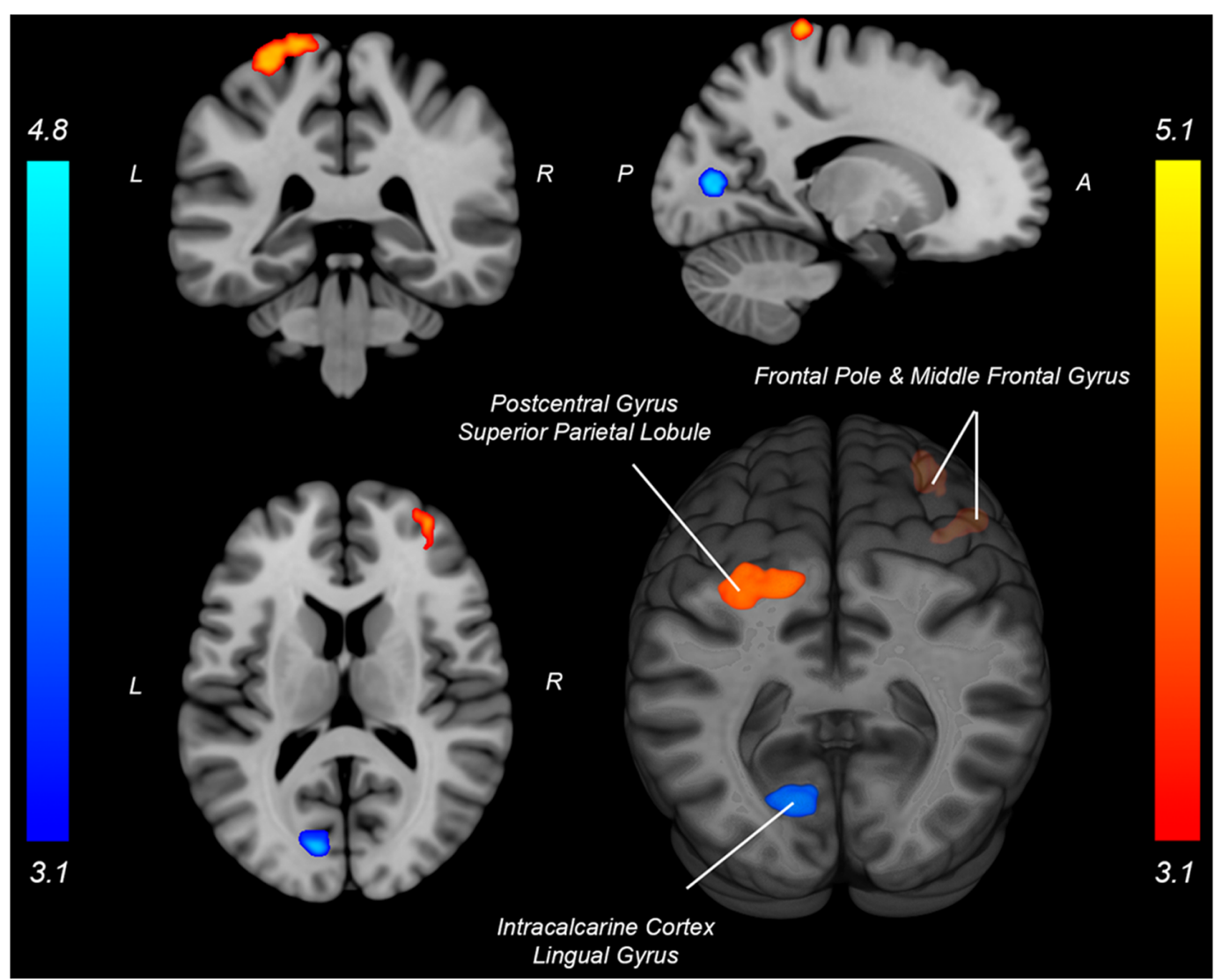

FIGURE 3 | Neural activity associated with knee extension force-matching error from Table 2 (Red: brain activity positively associated with an error. Blue: brain activity negatively associated with an error). P, posterior; A, anterior; L, left; R, right.

extrastriatal activity could also be secondary to visualizing good performance via alignment of the target and participant force and not be the mechanism for reduced error. As intracalcarine cortex and lingual gyrus have greater levels of activity when such crossmodal stimuli are congruent compared to incongruent stimuli (e.g., spatial and temporal correspondence of visual presentation and tactile stimulation) and low error results in a visual stimulus that is congruent with proprioceptive sensed force generation and tactile cues (Driver and Spence, 1998; Macaluso et al., 2000). 


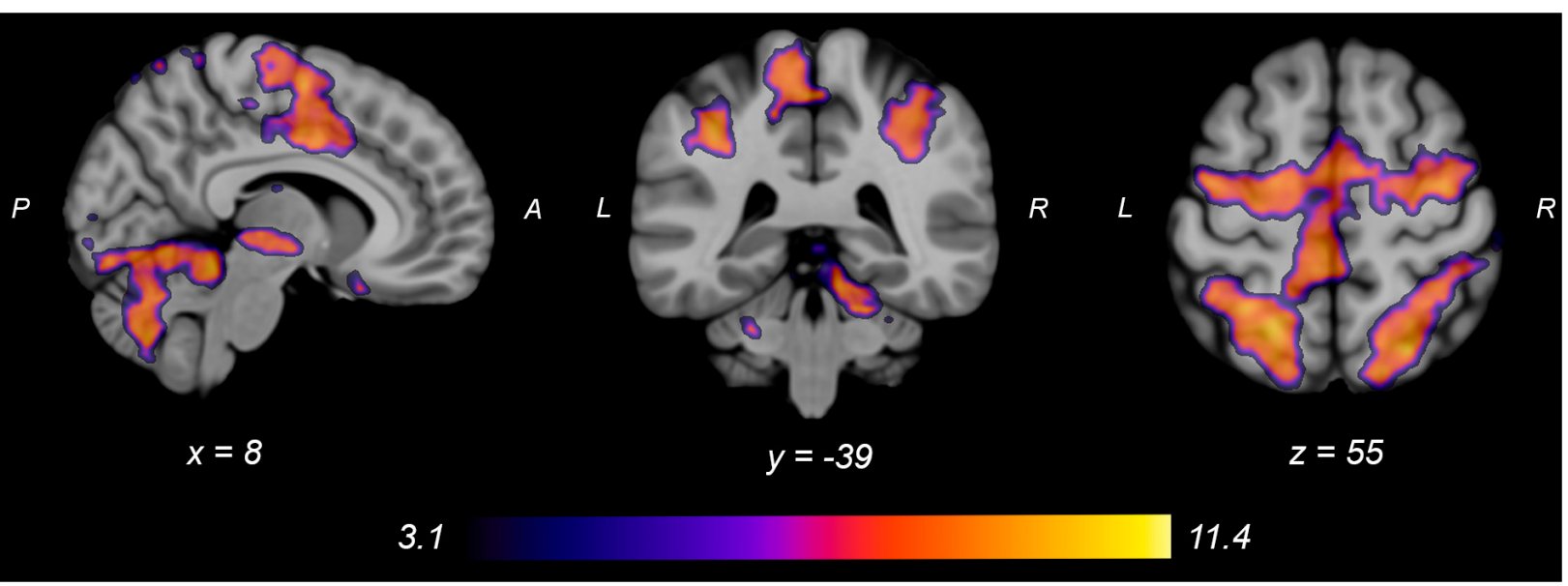

FIGURE 4 | Group average neural activity for knee flexion force-matching from Table 3. P, posterior; A, anterior; L, left; R, right.

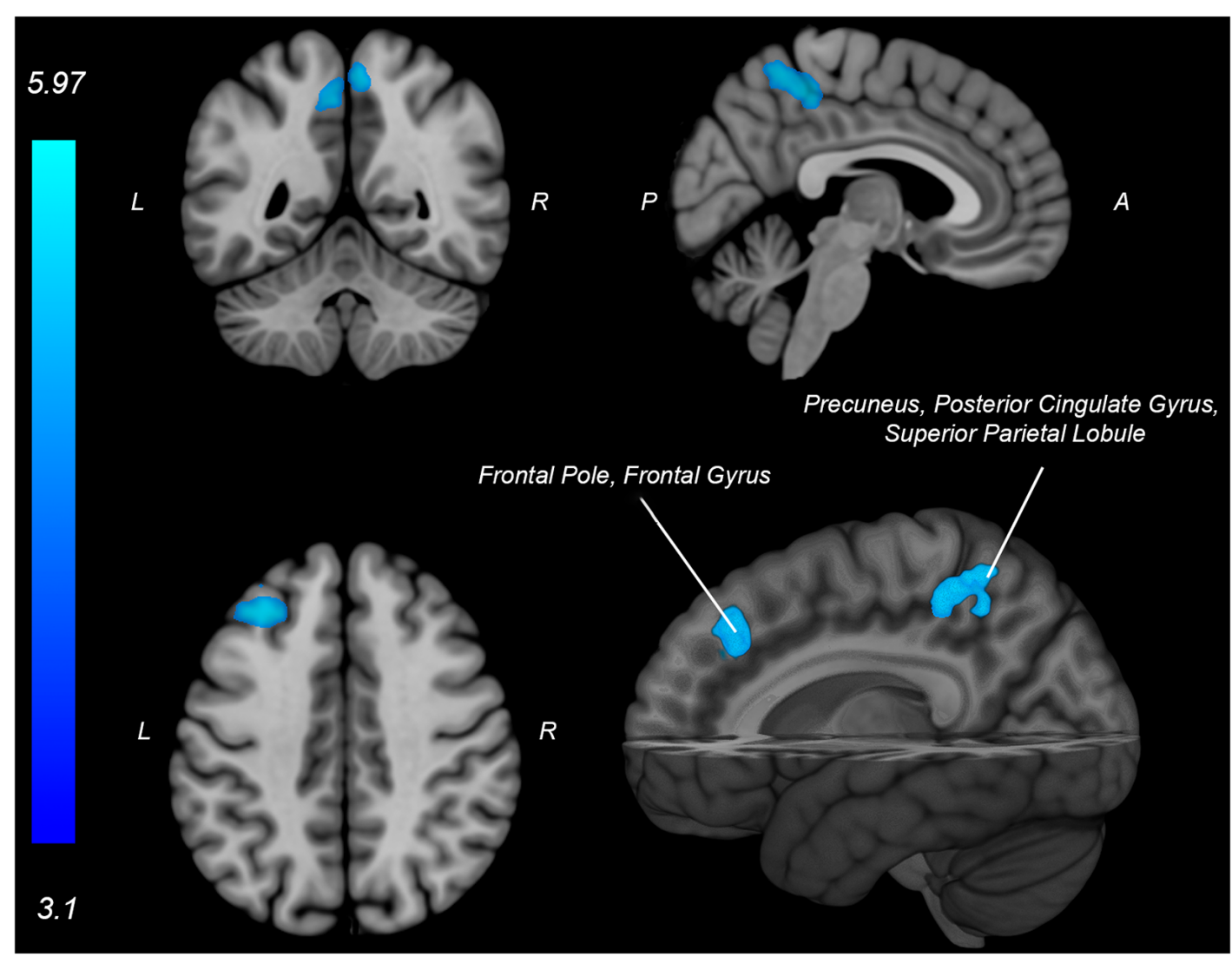

FIGURE 5 | Neural activity (blue) is negatively associated with knee flexion force-matching error from Table 3. P, posterior; A, anterior L, left; R, right.

\section{Neural Correlates of Knee Flexion Force Control}

The knee flexion force-matching task also had a neural activation pattern similar to prior lower extremity neuroimaging paradigms, with activation across the cortical and cerebellar sensorimotor network (Jaeger et al., 2014; Grooms et al., 2019). Knee flexion force error was associated with decreased activity in frontal and parietal regions, however, no increased neural activity was associated with knee flexion error. This contrasts with the knee extension force control error, which had increased 


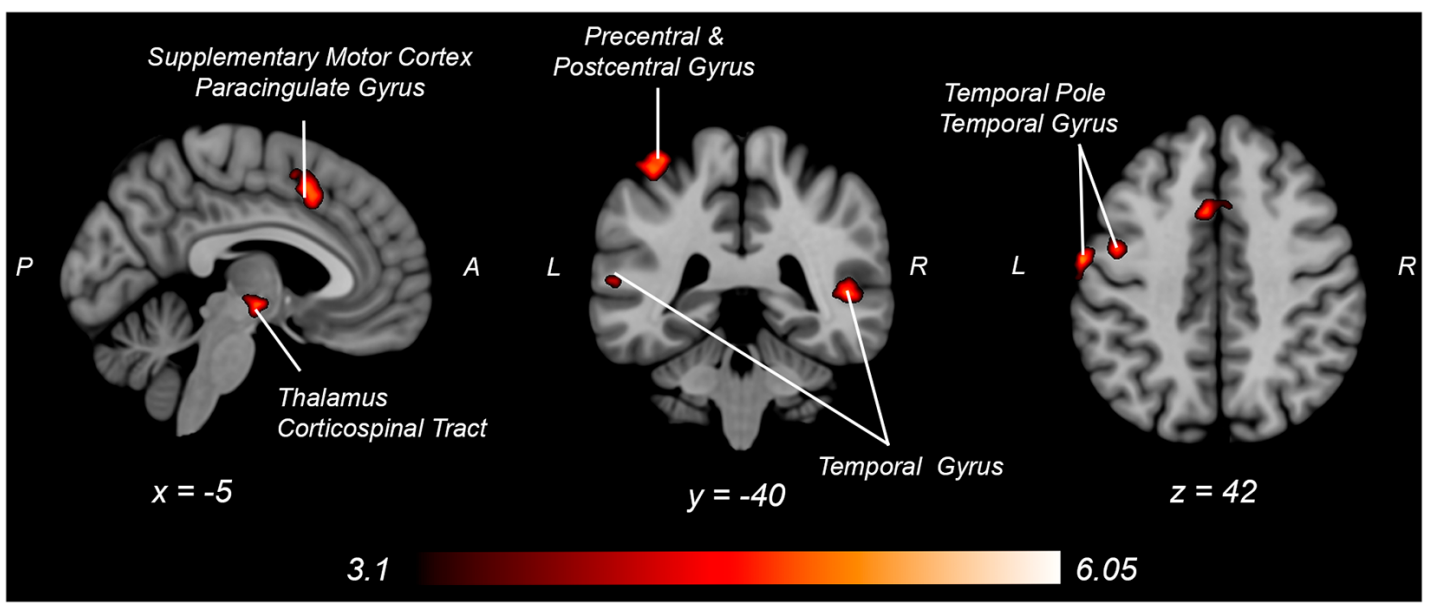

FIGURE 6 | Neural activity increases with knee flexion force matching relative to knee extension from Table 4. P, posterior; A, anterior L, left; R, right.

frontal and parietal lobe activity associated with increased force error.

This opposition may seem contradictory as one might expect more general alignment for the neural activity underlying force error between knee flexion and extension activities. However, the musculature enabling isolated knee flexion (primarily hamstring) vs. isolated knee extension (primarily quadriceps) have unique neural representation, peripheral nerve innervation, and spinal reflex structure (Jennings and Seedhom, 1994; MrachaczKersting et al., 2006). Thus, the brain differences for error correction between knee extension and flexion may be secondary to mediation at the spinal level. The hamstrings are also typically weaker than the quadriceps (Wyatt and Edwards, 1981; Aagaard et al., 1995; Pincivero et al., 1997) and have a greater proprioceptive error (Relph and Herrington, 2016), potentially secondary to decreased relative cortical representation (Davies, 2020) and less muscle spindle innervation relative to the quadriceps (Banks, 2006).

Anecdotally, the participants in this study had a more difficult time learning how to perform the knee flexion task relative to the knee extension task as many needed more practice trials for the hamstring task than the quadriceps task to achieve reliable performance. The constrained action hypothesis posits that when you attend to a motor task, you constrain the automatic, implicit motor programs that would have otherwise facilitated the movement (Wulf et al., 2001; Kal et al., 2013; Vidal et al., 2018). However, if there is no automatic, implicit motor plan present to guide the movement, then attention to the motor task may improve performance. Therefore, the knee extension task may have been more "intuitive" (implicit) in this sample, contributing to decreased frontal cortex activity not constraining the automatic motor program and facilitating reduced extension error. Conversely, if the knee flexion task is anecdotally less implicit (lacking a wellestablished, implicit motor program), the association between increased frontal activity and improved performance for flexion may be attributable to the necessity of cognitive-attentive neural processes to drive the motor plan.

\section{Neural Activity Differences Between Knee Extension and Flexion Force Control}

Engaging in knee flexion force control required increased cortical and subcortical activation, including primary sensorimotor cortex, secondary motor cortex, temporal regions, parietal supramarginal gyrus, and corticospinal tract, whereas no brain regions had increased activity for relative knee extension force control. These findings may partially explain the apparent paradoxical similar activation pattern associated with increased knee extension force error, yet decreased knee flexion force error, as the knee flexion force-matching task required greater overall neural activity for similar force-matching performance. This could be secondary to the relatively greater demand on the hamstrings, as they are typically weaker than the quadriceps, requiring elevated neural activity to produce the same force level. Alternatively, the position of the knee may have influenced the result as a near-terminal extension may bias toward quadriceps shortened position and improved steadiness (Krishnan et al., 2011) compared to the hamstring position (lengthened). A likely neurophysiologic contributor is the relatively increased spinal reflexive innervation of the hamstring (Shahani and Young, 1971; Roy et al., 2014; Mackey et al., 2016) requiring increased cortical activity to overcome potential spinal inhibition. The increased knee flexor force-matching neural activity could also be secondary to the task being more atypical, as concentric precise force control of the hamstrings is not as common to be engaged during locomotion, where the quadriceps is primarily engaged in concentric positioning and the hamstrings act eccentrically to decelerate before heel strike. Thus, the nature of the concentric force matching task may result in increased activation for knee flexion that would not be the case with an eccentric force-matching task (Koohestani et al., 2020). 


\section{LIMITATIONS}

This investigation was limited to a single joint position and an isometric contraction at a low force level, primarily to minimize head motion for fMRI. Possibly, synergist muscle groups that contribute to hip flexion or extension may reduce the ability to isolate the quadriceps for knee extension or the hamstring for knee flexion (though at the low force level required in this study, accessory muscle activity is unlikely), so future work may consider recording electromyography measures to ensure muscle group contributions. The force level was selected to ensure a sufficient fluctuation range to test force-sense but also keep head motion minimal. We used a low absolute value of $5 \mathrm{~N}$, as opposed to a low relative force such as $5 \%$ of a maximal voluntary contraction. Prior works have employed both a $\sim 5 \mathrm{~N}$ absolute threshold (Newton et al., 2008) and similar relative thresholds (Shanahan et al., 2015). As our sample was homogeneous in terms of fitness, activity level, age, and BMI, there is a minimal indication the results would be different if scaled to a relative $\%$ for capability. Nonetheless, future work across varied samples may consider employing a relative metric for the force target. While we enrolled young and physically active females to better understand knee force control in this population at a unique high-risk for sensorimotor-related coordination errors that contribute to knee ligament injuries such as the anterior cruciate ligament, our participant selection criteria limit generalization to males or aging populations. Future investigations may consider heterogenous demographical recruitment of participants to increase generalizability or determine if changes in neural activity are present with various ages or pathological populations. Additionally, the use of a variety of joint angles and intensities may also highlight how limb position and magnitude plays a role in central mechanisms of force regulation.

\section{CONCLUSION}

This investigation employed a novel lower extremity forcematching neuroimaging-compatible paradigm to examine motor control of the knee extensors and flexors. The paradigm was found to activate the sensorimotor network with unique neural correlates to force-matching error across parietal and frontal regions. This paradigm may allow for future research to better understand the neural correlates of lower extremity neuromuscular control across varied

\section{REFERENCES}

Aagaard, P., Simonsen, E. B., Trolle, M., Bangsbo, J., and Klausen, K. (1995). Isokinetic hamstring/quadriceps strength ratio: influence from joint angular velocity, gravity correction and contraction mode. Acta Physiol. Scand. 154, 421-427. doi: 10.1111/j.1748-1716.1995.tb09927.x

Abourezk, M. N., Ithurburn, M. P., McNally, M. P., Thoma, L. M., Briggs, M. S., Hewett, T. E., et al. (2017). Hamstring strength asymmetry at 3 years after anterior cruciate ligament reconstruction alters knee mechanics during gait and jogging. Am. J. Sports Med. 45, 97-105. doi: 10.1177/0363546516664705

Arhos, E. K., Thoma, L. M., Grindem, H., Logerstedt, D., Risberg, M. A., and Snyder-Mackler, L. (2020). Association of quadriceps strength symmetry and surgical status with clinical osteoarthritis 5 years after anterior cruciate pathologies or interventions. Specifically, this foundational work can support a future investigation into the unique contribution of the nervous system to lower extremity force regulation in pathologies that disrupt proprioception and sensorimotor function such as knee anterior cruciate ligament injury (Laboute et al., 2019), osteoarthritis (Shanahan, 2015), and patella-femoral pain (Te et al., 2017). As the evidence base for the role of the nervous system in these musculoskeletal conditions grows, the need for such paradigms that bridge neural activity and motor performance of the knee as described here are needed to provide pathology specific therapeutic targets (Silfies et al., 2017; Armijo-Olivo, 2018).

\section{DATA AVAILABILITY STATEMENT}

The raw data supporting the conclusions of this article will be made available by the authors, without undue reservation.

\section{ETHICS STATEMENT}

The studies involving human participants were reviewed and approved by Ohio University Institutional Review Board. The patients/participants provided their written informed consent to participate in this study.

\section{AUTHOR CONTRIBUTIONS}

DG, CC, and TW: conception. DG, CC, JS, and TW: experimental design and data analysis. DG, CC, AH, and TW: data collection. DG, CC, JS, AH, and TW: writing and review. All authors contributed to the article and approved the submitted version.

\section{FUNDING}

This study was supported by the US Department of Defense Congressionally Directed Medical Research Program Peer Reviewed Orthopaedic Research Program. Research Award (W81XWH-18-1-0707). Opinions, interpretations, conclusions, and recommendations are those of the author and are not necessarily endorsed by the Department of Defense.

ligament rupture. Arthritis Care Res. doi: 10.1002/acr.24479 [Epub ahead of print].

Armijo-Olivo, S. (2018). A new paradigm shift in musculoskeletal rehabilitation: why we should exercise the brain? Braz. J. Phys. Ther. 22, 95-96. doi: 10.1016/j. bjpt.2017.12.001

Ashe, J. (1997). Force and the motor cortex. Behav. Brain Res. 87, 255-269. doi: 10.1016/s0166-4328(97)00752-3

Astafiev, S. V., Stanley, C. M., Shulman, G. L., and Corbetta, M. (2004). Extrastriate body area in human occipital cortex responds to the performance of motor actions. Nat. Neurosci. 7, 542-548. doi: 10.1038/nn1241

Banks, R. W. (2006). An allometric analysis of the number of muscle spindles in mammalian skeletal muscles. J. Anat. 208, 753-768. doi: 10.1111/j.1469-7580. 2006.00558.x 
Baumeister, J., Reinecke, K., Schubert, M., and Weiss, M. (2011). Altered electrocortical brain activity after ACL reconstruction during force control. J. Orthop. Res. 29, 1383-1389. doi: 10.1002/jor.21380

Baweja, H. S., Patel, B. K., Martinkewiz, J. D., Vu, J., and Christou, E. A. (2009). Removal of visual feedback alters muscle activity and reduces force variability during constant isometric contractions. Exp. Brain Res. 197, 35-47. doi: 10.1007/s00221-009-1883-5

Beynnon, B. D., Vacek, P. M., Newell, M. K., Tourville, T. W., Smith, H. C., Shultz, S. J., et al. (2014). The effects of level of competition, sport, and sex on the incidence of first-time noncontact anterior cruciate ligament injury. Am. J. Sports Med. 42, 1806-1812. doi: 10.1177/03635465145 40862

Blackburn, T., Pietrosimone, B., Goodwin, J., and Johnston, C. (2017). Quadriceps/Hamstrings co-activation during gait in individuals with anterior cruciate ligament reconstruction. Osteoarthritis Cartilage 25, S110-S111. doi: 10.1016/j.joca.2017.02.178

Calvert, G. A. (2001). Crossmodal processing in the human brain: insights from functional neuroimaging studies. Cereb. Cortex 11, 1110-1123. doi: $10.1093 /$ cercor/11.12.1110

Davies, J. L. (2020). Using transcranial magnetic stimulation to map the cortical representation of lower-limb muscles. Clin. Neurophysiol. Pract. 5, 87-99. doi: $10.1016 /$ j.cnp.2020.04.001

Desikan, R. S., Ségonne, F., Fischl, B., Quinn, B. T., Dickerson, B. C., Blacker, D., et al. (2006). An automated labeling system for subdividing the human cerebral cortex on MRI scans into gyral based regions of interest. NeuroImage 31, 968-980. doi: 10.1016/j.neuroimage.2006.01.021

Diedrichsen, J., Balsters, J. H., Flavell, J., Cussans, E., and Ramnani, N. (2009). A probabilistic MR atlas of the human cerebellum. NeuroImage 46, 39-46. doi: 10.1016/j.neuroimage.2009.01.045

Docherty, C. L., and Arnold, B. L. (2008). Force sense deficits in functionally unstable ankles. J. Orthop. Res. 26, 1489-1493. doi: 10.1002/jor.20682

Driver, J., and Spence, C. (1998). Attention and the crossmodal construction of space. Trends Cogn. Sci. 2, 254-262. doi: 10.1016/S1364-6613(98) 01188-7

Dunst, B., Benedek, M., Jauk, E., Bergner, S., Koschutnig, K., Sommer, M., et al. (2014). Neural efficiency as a function of task demands. Intelligence 42, 22-30. doi: 10.1016/j.intell.2013.09.005

Eickhoff, S. B., Heim, S., Zilles, K., and Amunts, K. (2006). Testing anatomically specified hypotheses in functional imaging using cytoarchitectonic maps. NeuroImage 32, 570-582. doi: 10.1016/j.neuroimage.2006.04.204

Eickhoff, S. B., Paus, T., Caspers, S., Grosbras, M.-H., Evans, A. C., Zilles, K., et al. (2007). Assignment of functional activations to probabilistic cytoarchitectonic areas revisited. NeuroImage 36, 511-521. doi: 10.1016/j.neuroimage.2007. 03.060

Friston, K. J., Frith, C. D., Turner, R., and Frackowiak, R. S. (1995). Characterizing evoked hemodynamics with fMRI. NeuroImage 2, 157-165. doi: 10.1006/nimg. 1995.1018

Georgopoulos, A. P., Ashe, J., Smyrnis, N., and Taira, M. (1992). The motor cortex and the coding of force. Science 256, 1692-1695. doi: 10.1126/science.256. 5064.1692

Goense, J. B. M., and Logothetis, N. K. (2008). Neurophysiology of the BOLD fMRI signal in awake monkeys. Curr. Biol. 18, 631-640. doi: 10.1016/j.cub.2008. 03.054

Grooms, D. R., Diekfuss, J. A., Ellis, J. D., Yuan, W., Dudley, J., Foss, K. D. B., et al. (2019). A novel approach to evaluate brain activation for lower extremity motor control. J. Neuroimaging 29, 580-588. doi: 10.1111/jon. 12645

Hohmann, E., Tetsworth, K., and Glatt, V. (2019). The hamstring/quadriceps ratio is an indicator of function in ACL-deficient, but not in ACL-reconstructed knees. Arch. Orthop. Trauma Surg. 139, 91-98. doi: 10.1007/s00402-018 $-3000-3$

Hortobágyi, T., Garry, J., Holbert, D., and Devita, P. (2004). Aberrations in the control of quadriceps muscle force in patients with knee osteoarthritis. Arthritis Rheum. 51, 562-569. doi: 10.1002/art.20545

Jaeger, L., Marchal-Crespo, L., Wolf, P., Luft, A. R., Riener, R., Michels, L., et al. (2016). On the modulation of brain activation during simulated weight bearing in supine gait-like stepping. Brain Topogr. 29, 193-205. doi: 10.1007/s10548015-0441-7
Jaeger, L., Marchal-Crespo, L., Wolf, P., Riener, R., Michels, L., and Kollias, S. (2014). Brain activation associated with active and passive lower limb stepping. Front. Hum. Neurosci. 8:828. doi: 10.3389/fnhum.2014. 00828

Jenkinson, M., Bannister, P., Brady, M., and Smith, S. (2002). Improved optimization for the robust and accurate linear registration and motion correction of brain images. NeuroImage 17, 825-841. doi: 10.1016/s10538119(02)91132-8

Jenkinson, M., Beckmann, C. F., Behrens, T. E. J., Woolrich, M. W., and Smith, S. M. (2012). FSL. NeuroImage 62, 782-790. doi: 10.1016/j.neuroimage. 2011.09.015

Jennings, A. G., and Seedhom, B. B. (1994). Proprioception in the knee and reflex hamstring contraction latency. J. Bone Joint Surg. Br. 76, 491-494. doi: 10.1302/0301-620x.76b3.8175863

Kal, E. C., van der Kamp, J., and Houdijk, H. (2013). External attentional focus enhances movement automatization: a comprehensive test of the constrained action hypothesis. Hum. Mov. Sci. 32, 527-539. doi: 10.1016/j.humov.2013. 04.001

Kapreli, E., Athanasopoulos, S., Papathanasiou, M., Van Hecke, P., Kelekis, D., Peeters, R., et al. (2007). Lower limb sensorimotor network: issues of somatotopy and overlap. Cortex 43, 219-232. doi: 10.1016/s00109452(08)70477-5

Kapreli, E., Athanasopoulos, S., Papathanasiou, M., Van Hecke, P., Strimpakos, N., Gouliamos, A., et al. (2006). Lateralization of brain activity during lower limb joints movement. An fMRI study. NeuroImage 32, 1709-1721. doi: 10.1016/j. neuroimage.2006.05.043

Koohestani, M., Sharifnezhad, A., Abbasi, A., Javadi, A.-H., and Gokeler, A. (2020). Brain activation during maximum concentric and eccentric knee extension muscle contractions. J. Brain Nerves 2020, 1-6. doi: 10.31487/j.jbn. 2020.01.05

Kriegeskorte, N., Lindquist, M. A., Nichols, T. E., Poldrack, R. A., and Vul, E. (2010). Everything you never wanted to know about circular analysis, but were afraid to ask. J. Cereb. Blood Flow Metab. 30, 1551-1557. doi: 10.1038/jcbfm. 2010.86

Kriegeskorte, N., Simmons, W. K., Bellgowan, P. S. F., and Baker, C. I. (2009). Circular analysis in systems neuroscience: the dangers of double dipping. Nat. Neurosci. 12, 535-540. doi: 10.1038/nn.2303

Krishnan, C., Allen, E. J., and Williams, G. N. (2011). Effect of knee position on quadriceps muscle force steadiness and activation strategies. Muscle Nerve 43 , 563-573. doi: 10.1002/mus.21981

Laboute, E., Verhaeghe, E., Ucay, O., and Minden, A. (2019). Evaluation kinaesthetic proprioceptive deficit after knee anterior cruciate ligament (ACL) reconstruction in athletes. J. Exp. Ortop. 6:6. doi: 10.1186/s40634-019 $-0174-8$

Liu, J. Z., Dai, T. H., Elster, T. H., Sahgal, V., Brown, R. W., and Yue, G. H. (2000). Simultaneous measurement of human joint force, surface electromyograms, and functional MRI-measured brain activation. J. Neurosci. Methods 101, 49-57. doi: 10.1016/s0165-0270(00)00252-1

Logothetis, N. K., Pauls, J., Augath, M., Trinath, T., and Oeltermann, A. (2001). Neurophysiological investigation of the basis of the fMRI signal. Nature 412, 150-157. doi: $10.1038 / 35084005$

Luft, A. R., Smith, G. V., Forrester, L., Whitall, J., Macko, R. F., Hauser, T.-K., et al. (2002). Comparing brain activation associated with isolated upper and lower limb movement across corresponding joints. Hum. Brain Mapp. 17, 131-140. doi: $10.1002 / \mathrm{hbm} .10058$

Macaluso, E., Frith, C. D., and Driver, J. (2000). Modulation of human visual cortex by crossmodal spatial attention. Science 289, 1206-1208. doi: $10.1126 /$ science.289.5482.1206

Mackey, A. S., Uttaro, D., McDonough, M. P., Krivis, L. I., and Knikou, M. (2016). Convergence of flexor reflex and corticospinal inputs on tibialis anterior network in humans. Clin. Neurophysiol. 127, 706-715. doi: 10.1016/j.clinph. 2015.06.011

Manini, T. M., and Clark, B. C. (2012). Dynapenia and aging: an update. J. Gerontol. A Biol Sci. Med. Sci. 67A, 28-40. doi: 10.1093/gerona/glr010

Manini, T. M., Visser, M., Won-Park, S., Patel, K. V., Strotmeyer, E. S., Chen, H., et al. (2007). Knee extension strength cutpoints for maintaining mobility. J. Am. Geriatr. Soc. 55, 451-457. doi: 10.1111/j.1532-5415.2007. 01087.x 
Marx, R. G., Stump, T. J., Jones, E. C., Wickiewicz, T. L., and Warren, R. F. (2001). Development and evaluation of an activity rating scale for disorders of the knee. Am. J. Sports Med. 29, 213-218. doi: 10.1177/03635465010290 021601

McGregor, K. M., Carpenter, H., Kleim, E., Sudhyadhom, A., White, K. D., Butler, A. J., et al. (2012). Motor map reliability and aging: a TMS/fMRI study. Exp. Brain Res. 219, 97-106. doi: 10.1007/s00221-0123070-3

Mehta, J. P., Verber, M. D., Wieser, J. A., Schmit, B. D., and Schindler-Ivens, S. M. (2009). A novel technique for examining human brain activity associated with pedaling using fMRI. J. Neurosci. Methods 179, 230-239. doi: 10.1016/j. jneumeth.2009.01.029

Mehta, J. P., Verber, M. D., Wieser, J. A., Schmit, B. D., and SchindlerIvens, S. M. (2012). The effect of movement rate and complexity on functional magnetic resonance signal change during pedaling. Motor Control 16, 158-175. doi: $10.1123 /$ mcj.16.2.158

Montalvo, A. M., Schneider, D. K., Yut, L., Webster, K. E., Beynnon, B., Kocher, M. S., et al. (2019). "What's my risk of sustaining an ACL injury while playing sports?” A systematic review with meta-analysis. Br. J. Sports Med. 53, 1003-1012. doi: 10.1136/bjsports-2016-096274

Mrachacz-Kersting, N., Grey, M. J., and Sinkjær, T. (2006). Evidence for a supraspinal contribution to the human quadriceps long-latency stretch reflex. Exp. Brain Res. 168, 529-540. doi: 10.1007/s00221-005-0120-0

Naufel, S., Glaser, J. I., Kording, K. P., Perreault, E. J., and Miller, L. E. (2019). A muscle-activity-dependent gain between motor cortex and EMG. J. Neurophysiol. 121, 61-73. doi: 10.1152/jn.00329.2018

Newton, J. M., Dong, Y., Hidler, J., Plummer-D’Amato, P., Marehbian, J., Albistegui-Dubois, R. M., et al. (2008). Reliable assessment of lower limb motor representations with fMRI: use of a novel MR compatible device for real-time monitoring of ankle, knee and hip torques. NeuroImage 43, 136-146. doi: 10.1016/j.neuroimage.2008.07.001

Pincivero, D. M., Lephart, S. M., and Karunakara, R. G. (1997). Relation between open and closed kinematic chain assessment of knee strength and functional performance. Clin. J. Sport Med. 7, 11-16. doi: 10.1097/00042752-19970100000003

Poldrack, R. A., Mumford, J. A., and Nichols, T. E. (2011). Handbook of Functional MRI Data Analysis [Internet]. Cambridge: Cambridge University Press.

Poortvliet, P. C., Tucker, K. J., Finnigan, S., Scott, D., Sowman, P., and Hodges, P. W. (2015). Cortical activity differs between position- and force-control knee extension tasks. Exp. Brain Res. 233, 3447-3457. doi: $10.1007 / \mathrm{s} 00221-015-4404-8$

Relph, N., and Herrington, L. (2016). The effects of knee direction, physical activity and age on knee joint position sense. Knee 23, 393-398. doi: 10.1016/j.knee. 2016.02.018

Roy, F. D., Bosgra, D., and Stein, R. B. (2014). Interaction of transcutaneous spinal stimulation and transcranial magnetic stimulation in human leg muscles. Exp. Brain Res. 232, 1717-1728. doi: 10.1007/s00221-014 $-3864-6$

Schubotz, R. I., and von Cramon, D. Y. (2002). A blueprint for target motion: fMRI reveals perceived sequential complexity to modulate premotor cortex. NeuroImage 16, 920-935. doi: 10.1006/nimg.2002.1183

Shahani, B. T., and Young, R. R. (1971). Human flexor reflexes. J. Neurol. Neurosurg. Psychiatry 34, 616-627. doi: 10.1136/jnnp.34.5.616

Shanahan, C. (2015). Sensorimotor Changes in Knee Osteoarthritis: From Muscle Spindle Function to Brain Organisation and Activity. Available online at: http://minerva-access.unimelb.edu.au/handle/11343/91673. Accessed November 2, 2018

Shanahan, C. J., Hodges, P. W., Wrigley, T. V., Bennell, K. L., and Farrell, M. J. (2015). Organisation of the motor cortex differs between people with and without knee osteoarthritis. Arthritis Res. Ther. 17:164. doi: 10.1186/s13075015-0676-4
Silfies, S. P., Vendemia, J. M. C., Beattie, P. F., Stewart, J. C., and Jordon, M. (2017). Changes in brain structure and activation may augment abnormal movement patterns: an emerging challenge in musculoskeletal rehabilitation. Pain Med. 18, 2051-2054. doi: 10.1093/pm/pnx190

Smith, S. M., Jenkinson, M., Woolrich, M. W., Beckmann, C. F., Behrens, T. E. J., Johansen-Berg, H., et al. (2004). Advances in functional and structural MR image analysis and implementation as FSL. NeuroImage 23, S208-S219. doi: 10.1016/j.neuroimage.2004.07.051

Te, M., Baptista, A. F., Chipchase, L. S., and Schabrun, S. M. (2017). Primary motor cortex organization is altered in persistent patellofemoral pain. Pain Med. 18, 2224-2234. doi: 10.1093/pm/pnx036

Telianidis, S., Perraton, L., Clark, R. A., Pua, Y.-H., Fortin, K., and Bryant, A. L. (2014). Diminished sub-maximal quadriceps force control in anterior cruciate ligament reconstructed patients is related to quadriceps and hamstring muscle dyskinesia. J. Electromyogr. Kinesiol. 24, 513-519. doi: 10.1016/j.jelekin.2014. 04.014

Tourville, T. W., Jarrell, K. M., Naud, S., Slauterbeck, J. R., Johnson, R. J., and Beynnon, B. D. (2014). Relationship between isokinetic strength and tibiofemoral joint space width changes after anterior cruciate ligament reconstruction. Am. J. Sports Med. 42, 302-311. doi: $10.1177 / 0363546513510672$

Tracy, B. L. (2007). Visuomotor contribution to force variability in the plantarflexor and dorsiflexor muscles. Hum. Mov. Sci. 26, 796-807. doi: 10.1016/j.humov.2007.07.001

Tracy, B. L., Dinenno, D. V., Jorgensen, B., and Welsh, S. J. (2007). Aging, visuomotor correction, and force fluctuations in large muscles. Med. Sci. Sports Exerc. 39, 469-479. doi: 10.1249/mss.0b013e31802d3ad3

Trinastic, J. P., Kautz, S. A., McGregor, K., Gregory, C., Bowden, M., Benjamin, M. B., et al. (2010). An fMRI study of the differences in brain activity during active ankle dorsiflexion and plantarflexion. Brain Imaging Behav. 4, 121-131. doi: 10.1007/s11682-010-9091-2

Vidal, A., Wu, W., Nakajima, M., and Becker, J. (2018). Investigating the constrained action hypothesis: a movement coordination and coordination variability approach. J. Mot. Behav. 50, 528-537. doi: 10.1080/00222895.2017. 1371111

Ward, N. S., Swayne, O. B. C., and Newton, J. M. (2008). Age-dependent changes in the neural correlates of force modulation: an fMRI study. Neurobiol. Aging 29, 1434-1446. doi: 10.1016/j.neurobiolaging.2007. 04.017

Ward, S. H., Perraton, L., Bennell, K., Pietrosimone, B., and Bryant, A. L. (2019). Deficits in quadriceps force control after anterior cruciate ligament injury: potential central mechanisms. J. Athl. Train. 54, 505-512. doi: 10.4085/10626050-414-17

Wulf, G., McNevin, N., and Shea, C. H. (2001). The automaticity of complex motor skill learning as a function of attentional focus. Q. J. Exp. Psychol. A 54, 1143-1154. doi: 10.1080/713756012

Wyatt, M. P., and Edwards, A. M. (1981). Comparison of quadriceps and hamstring torque values during isokinetic exercise. J. Orthop. Sports Phys. Ther. 3, 48-56. doi: 10.2519/jospt.1981.3.2.48

Conflict of Interest: The authors declare that the research was conducted in the absence of any commercial or financial relationships that could be construed as a potential conflict of interest.

Copyright $\odot 2021$ Grooms, Criss, Simon, Haggerty and Wohl. This is an open-access article distributed under the terms of the Creative Commons Attribution License (CC BY). The use, distribution or reproduction in other forums is permitted, provided the original author(s) and the copyright owner(s) are credited and that the original publication in this journal is cited, in accordance with accepted academic practice. No use, distribution or reproduction is permitted which does not comply with these terms. 